\title{
Further Developments in Systems Biology
}

\section{G W Ewing*}

Montague Healthcare, Mulberry House, 6 Vine Farm Close, Cotgrave, Nottingham NG12 3TU, United Kingdom

\begin{abstract}
There is a theoretical void between cognition and cellular \& molecular biology. Most biomedical research focuses upon the exome0[-p i.e. of proteins expressed (genotype) and almost completely ignores phenotype however $90 \%$ of drugs, which are based upon drug-protein interactions, are recognised to be ineffective in circa $50 \%$ of the population i.e. circa $50 \%$ is unexplained]. Factors such as age, weight, sex, racial origins, the influence and nature of stress, etc; influence the way in which drugs function. In order to explain this identified theoretical void research must take into account the cognitive or environmental factors which influence phenotype and which influence the ability of proteins to react e.g. $\mathrm{pH}$; temperature; levels of minerals, hormones, cofactors; blood glucose; blood cell content; digestion; excretion/elimination of toxins; osmotic pressure; sleep; breathing; etc. These are the physiological systems, the study of which is recognised to be significantly under-researched; perhaps because there are few tools which are available for researchers to use to research the physiological systems.
\end{abstract}

The physiological or functional systems comprise networks of organs which perform a unique physiological function. Under normal circumstances these systems are regulated by the neuroregulatory processes in the brain - neuroregulatory dysfunction leading to the impaired function of one organ and hence the instability of one or more organ networks.

Light offers a technique which can be used to make an assessment of genotype and phenotype. The energy of light absorbed by proteins being an expression of genotype and the energy of light emitted by proteins and pathologies being an expression of rate of reaction or phenotype. It enables researchers to differentiate between the measured levels of expressed proteins, the uncoiled and unreactive nature of proteins, and the rate at which proteins react. This is particularly significant in the case of diabetes mellitus in which altered colour perception is linked to the early onset of the condition and before the onset of diabetic retinopathy. Moreover the innate and extraordinary ability of the eye to detect small amounts of light indicates that this technique may be able to diagnose the early onset of pathologies earlier than orthodox biomedical techniques. This absorption and emission of biophotons is the biochemical equivalent of bits of information used in a computer. It deals with the rate at which proteins react rather than their levels. Accordingly it is not influenced by the limitations of contemporary biochemical testing and can diagnose the onset of pathologies from their presymptomatic origins.

Virtual Scanning is the first of a new generation of medical technology. It is based upon a mathematical model of the physiological systems. To be precise, it is based upon a mathematical model of the consequences of cognitive input (mainly, but not solely, of colour perception) upon the autonomic nervous system. It incorporates an understanding of the structure and significance of the organ networks commonly referred to as physiological systems. It provides a summary of genotype and phenotype for all organs and most common pathologies which can be hugely valuable (i) advancing the current level of understanding of disease states, (ii) in routine medical diagnosis and (iii) advancing the development of new drugs or therapies which can be used as treatments.

Keywords: Cognitive; Mathematical model; Physiological systems; Autonomic nervous system

\section{Introduction}

The study of Metabolite Profiles in Biological Systems is considered to be a significant issue for biomedicine however what do we mean when we consider what is a metabolite and what is a biological system? Metabolites are the product of protein reactions, and our proteins are expressed our genes so metabolite profiles must be considered along with the data derived from other disciplines.

Furthermore, many diseases are poorly defined. They are often polygenic. Furthermore the particular genes responsible for a disease can differ between racial sub-types [1]. They can have multiple causes and they often influence the function of more than one physiological or biological system e.g. the etiology of migraines includes factors which inhibit the delivery of oxygen to the brain (involving heart function, blood viscosity, delivery of blood to the lungs, the structure of the spine, neural blood-flow, etc).

The study of genetics considers the chemical structure of genes responsible for the expression of proteins however this can overlook the significance of genetic conformation or structure. This focus upon genotype ignores the immense influence of phenotype or of environmental factors including what we perceive as stress.

\section{Omics}

The study of proteomics identifies the nature and structure of proteins which are expressed by our genes (genomics). This overlooks the factors such as $\mathrm{pH}$ and temperature which influence the ability of a protein to coil or uncoil and hence react with its reactive substrate

${ }^{*}$ Corresponding author: G.W. Ewing, Director, Montague Healthcare, Mulberry House, 6 Vine Farm Close, Cotgrave, Nottingham NG12 3TU, United Kingdom, E-mail: graham.ewing@montaguehealthcare.co.uk

Received March 05, 2012; Accepted March 19, 2012; Published March 21, 2012

Citation: Ewing GW (2012) Further Developments in Systems Biology. Metabolomics S1:001. doi:10.4172/2153-0769.S1-001

Copyright: (c) 2012 Ewing GW. This is an open-access article distributed unde the terms of the Creative Commons Attribution License, which permits unrestricted use, distribution, and reproduction in any medium, provided the original author and source are credited. 
(proteomics) and which ultimately influences the level and spectrum of chemical metabolites (metabolomics).

Many proteins are visually active. They absorb and emit light. This absorbed and emitted light, more typically known as auto fluorescence of natural bioluminescence, influences our perception of colour. The spectrum of light absorbed and emitted alters with the emergence of pathologies i.e. light is a metabolite. For example the change of visual contrast and altered blue-yellow colour perception, which is commonly associated with type 2 diabetes, is associated with the glycation of proteins [2]. Moreover this occurs prior to the onset of diabetic retinopathy [3]. It is a direct measure of the rate of onset of type 2 diabetes from its presymptomatic onset.

The regulation of $\mathrm{pH}$ and temperature are two of the 13 neurally regulated physiological systems or organ networks which have been identified by Dr Igor Gennadyevich Grakov. Increased acidity and temperature alters the levels of minerals and protein conformation. This influences the rate of reaction, spectrum of metabolites, the frequency and intensity of light emitted, spatial distribution of proteins within the cell, and ultimately distorts cell morphology.

The physiological systems influence all physiologically significant reactions and extractions e.g. instead of a cardiovascular system there are instead other sub-systems: breathing, blood pressure, blood glucose, blood volume and blood cell content. I.G. Grakov has established the existence of 13 neurally regulated physiological systems: $\mathrm{pH}$, temperature; breathing, blood glucose, blood pressure, blood volume, blood cell content, osmotic pressure, digestion, elimination, sexual function, posture and sleep. This is a revised the orthodox definition of physiological systems. These physiological systems influence the rate of all physiologically significant reactions and extractions.

In principle it forms the basis of a cognitive technique known as Virtual Scanning which can be used to screen for the earliest emergence of pathologies, to determine the onset of pathologies and the influence of genotype and phenotype in each pathology, to monitor or predict changes to cell morphology, and stimulate or activate the function of less reactive or uncoiled proteins.

I.G. Grakov appears to have mathematically modelled the physiological systems i.e. he has established a fundamental relationship between cognition, in particular of visual perception of colours, and cellular and molecular biology [4]. This model incorporates an understanding of the nature, structure and significance of the neurally regulated physiological structures or systems. This programme of research which commenced at the University of Novosibirsk (circa 1980-2005/6) has been incorporated into a unique technology which may overcome the limitations of many scanning or biomarker techniques [5,6]. Such techniques ignore the influence of stress and environmental factors (phenotype). Furthermore each new tests adds to the burden, of time and cost, placed upon clinicians to accurately diagnose and treat disease. There is a market need for significantly better and cheaper means of diagnosing and treating disease. We posit the following questions:

1. Is there a gold standard against which a test can be compared?

2. Does the Does the condition have multiple origins?

3. Does it have genetic and environmental/phenotypic origins?

4. Can a single biomarker ever be an accurate measure in complex multi-systemic conditions where diseases can have more than one origin or outcome?
Consider also the effectiveness of drugs. 90\% of drugs are ineffective in $50 \%$ of the population [7]. Most drugs are based upon the interaction of the drug substance with a protein which has been identified to be part of the pathological process. If most drugs are only about $50 \%$ effective - What about the other $50 \%$ ?

Gene profiling alone is unable to explain the etiology of many diseases. Most diseases are influenced by a combination of genetic and environmental factors. For example in the case of the BRCA2 gene which is associated with increased risk of breast cancer, the onset of type 2 diabetes, the onset of angina pectoris and cardiovascular disease, the onset of asthma, depression, etc. It is perfectly plausible for a medical condition to be detected by a genetic test (genomics) but that its development is relatively benign until activated by a stressor i.e. by phenotype (phenomics). It is only the expression of proteins which is genetic. The factors which influence the ability of proteins to react are largely, but not entirely, environmental e.g.

i. lack of exercise contributes to increased acidity.

ii. increased levels of stress contributes to increased acidity.

iii. consumption of acidified beverages contributes to increased acidity which alters the prevailing levels of minerals and ultimately susceptibility to a ladder of cascade of pathologies - diabetes, heart conditions, circulatory problems, cancers, etc.

iv. the genetic profile can return to its premorbid state e.g. following an exercise programme [8].

This raises a number of significant questions (i) which factors influence the rate at which the genes express proteins? Known contributory factors include famine i.e. the supply of glucose, and drought? (ii) which factors influence the rate at which proteins react? (iii) which factors influence the coiled/uncoiled nature of proteins? The coiled or uncoiled nature of proteins (i) influences reaction outcomes; (ii) cell morphology; and (iii) is a significant factor in the emergence of diabetes, Alzheimer's disease, cystic fibrosis, etc.

Proteins are often visually active. In the case of diabetes glycated haemoglobin HbAlc is often used as a marker. Glycated proteins (e.g. albumin, haemoglobin, insulin, and LDL Cholesterol) are bioluminescent [2]. A number of tests have been developed to measure the bioluminescence of body fluids as a means of diagnosing diabetes.

This differentiation between genotype and phenotype enables us to look at a number of different scenarios in the disease process e.g.

i. there can be normal level of protein (or other biologically significant chemical) and it will react normally;

ii. there can be normal level of protein but it cannot react e.g. insulin resistance, uncoiled protein, changes to $\mathrm{pH}$, mineral insufficiency;

iii. there can be a low level of protein but it will react e.g. a patient can be diagnosed with anaemia yet shows no symptoms;

iv. there can be a low level of protein but it will not react;

v. there can be a low level of a protein or enzyme which is below the level necessary to sustain normal function e.g. Gaucher's disease, Fabry's disease, etc.

This enables us to explain false positives and false negatives i.e. in which a patient could be diagnosed with a medical condition yet would not display the symptoms and vice versa. 

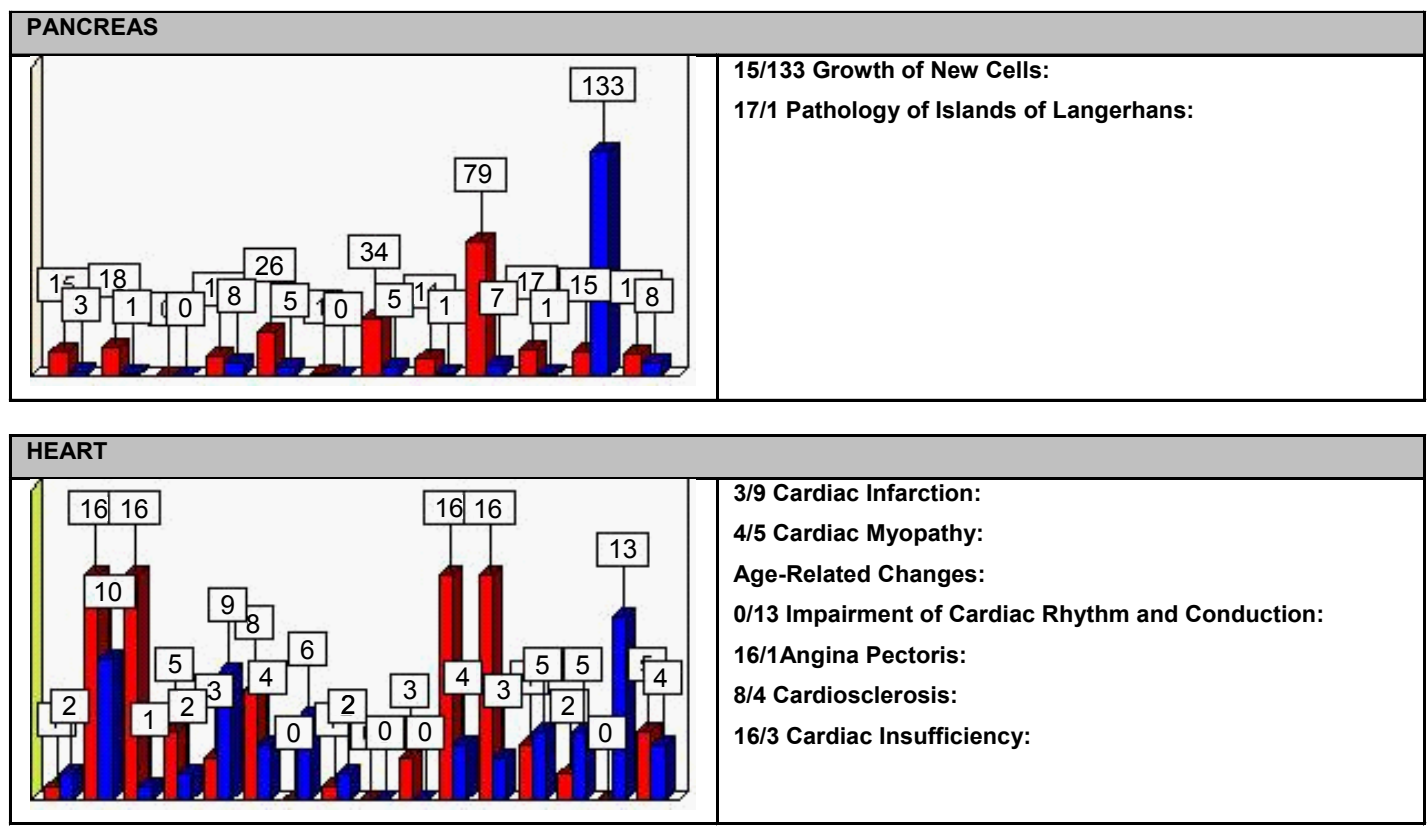

Figure 1:

The current method for diagnosing type 2 diabetes is to establish whether a patient's blood glucose results fall within an expected range of typically 4-8 m mol per litre. This presents problems because a patient's normal range will alter with age, racial sub-type, gender, and even throughout the month or day. What is required is a test which is essentially linear i.e. the greater the test result - the greater the degree of dysfunction or disease. Patients with type 2 diabetes have altered colour perception and this altered perception of blue and yellow occurs prior to the onset of diabetic retinopathy [3]. The colour spectrum and intensity of the light emitted i.e. of bio photon emission, which influences our perception of colour, is a metabolite and biomarker of each prevailing pathology.

Virtual Scanning is a cognitive, computer-based technology. It is a fluorescence-based methodology incorporating elements which are more typical of systems biology, bioinformatics, optometry, cognitive psychology, proteomics, genomics, metabolomics, etc.

The unique light absorbing and emitting properties of proteins influence visual perception e.g. diabetic pathologies (glycation) releases a proportionate amount of bio photons. This emission of light occurs prior to the onset of diabetic retinopathy i.e. at the earliest onset of the diabetic pathology.

\section{The Cognitive Approach/Virtual Scanning}

Virtual Scanning appears to have the potential of being the next major step forward in the evolution of diagnostic tests - the biomarker(s) being the colour spectrum and intensity of the light absorbed and emitted. In principle, it may offer earlier, better, more comprehensive, safer, faster and significantly less expensive means of diagnosing disease. It is a developed technology platform which has the ability to diagnose a wide range of medical conditions in an unprecedented level of detail and can also provide a pictorial representation or image of changes to cell morphology. It represents a number of issues which, although considered feasible by researchers, are relatively new medical concepts e.g.
- Top-down Systems Biology.

- Mathematical model of the consequences of cognition, in particular of visual perception upon the Autonomic Nervous System; which includes a revised understanding of the nature and structure of the physiological systems.

- Mathematical model of the physiological systems [9-11].

- Personal biological modelling.

Virtual Scanning is a cognitive technique which involves studying a short video and memorising the colour balance of the presented video. At the end of 15 seconds a colour filter is imposed. The task is to use the 'mouse' to select colours from the colour palette (at the lower side of the screen) and to recover the original colour balance. This provides the computer programme with the data sets for the mathematical programme. The computer considers the way which each person has recovered the picture, the speed of completing the task, the accuracy of their memory, etc. The results are presented typically as follows:

- Diagnose/screen the onset of circa 15 medical indications in each of circa 32-33 organs (see Figure 1)

- Determine the influence of genotype or phenotype in each medical indication

- Screen/image the extent of changes to cell morphology which are associated with each medical indication

- Predict the onset of further medical complications

This technique has been shown to have the potential to screen for the earliest emergence of diabetes [6], to screen for the earliest emergence of heart pathologies [12], to diagnose migraine [13], to contribute to a better understanding of developmental dyslexia $[14,15]$ etc.

The technology developed by Dr. Grakov, Virtual Scanning, was demonstrated at convenient opportunities throughout this conference. 


\section{Summary}

There is a need to better integrate genomic, proteomic, and metabolomic information to further understand the processes associated with the onset of pathologies. There is also a need to consider the influence which cognitive/sensory input has upon the autonomic nervous system; the nature and significance of the physiological systems; and the complex role of phenotype. The study of the light which is absorbed and emitted by proteins, which is incorporated into Virtual Scanning, may offer the means to do so.

\section{References}

1. Bodhini D, Radha V, Ghosh S, Majumder P, Mohan V (2011) Lack of association of PTPN1 gene polymorphisms with type 2 diabetes in south Indians. J Genet 90: $323-326$

2. Ewing GW, Parvez SH, Grakov IG (2011) Further Observations on Visual Perception: the influence of pathologies upon the absorption of light and emission of bioluminescence. Open Syst Biol J 4: 1-7.

3. Beisswenger PJ, Makita Z, Curphey TJ, Moore LL, Jean S, et al. (1995) Formation of immunochemical advanced glycosylation end products precedes and correlates with early manifestations of renal and retinal disease in diabetes. Diabetes 44: 824-829.

4. Ewing GW, Ewing EN (2008) Cognition, the Autonomic Nervous System and the Physiological Systems. J. Biogenic Amines 22: 140-163.

5. Ewing GW, Parvez SH (2010) The Dynamic Relationship between Cognition, the Physiological Systems, and Cellular and Molecular Biochemistry: a Systems-based Perspective on the Processes of Pathology. Act Nerv Super Rediviva 52: 29-36.
6. Ewing GW, Parvez SH (2010) The Multi-systemic Nature of Diabetes Mellitus: genotype or phenotype? N Am J Med Sci 2: 444-456.

7. Spear BB, Heath-Chiozzi M, Huff J (2001) Clinical Applications of Pharmacogenetics. Trends in Molecular Medicine 7: 201-204.

8. Booth FW, Neufer PD (2005). Exercise Controls Gene Expression. American Scientist 93: 28-35

9. Ewing GW (2010) Mathematical Modeling the Neuroregulation of Blood Pressure using a Cognitive Top-down Approach. N Am J Med Sci 2: 341-352.

10. Ewing GW, Parvez SH (2011) Mathematical Modeling the Systemic Regulation of Blood Glucose: ‘a top-down' Systems Biology Approach. Neuro Endocrino Lett 32: 371-379

11. Ewing GW. The Regulation of $\mathrm{pH}$ is a Physiological System. Increased Acidity alters Protein Conformation and Cell Morphology and is a Significant Facto in the onset of Diabetes and other common pathologies. The Open Systems Biology Journal: in press.

12. Ewing GW, Ewing EN (2009) Computer Diagnosis in Cardiology. N Am J Med Sci 1: 152-159.

13. Ewing GW, Ewing EN, Parvez SH (2009) The Multi-systemic Origins of Migraine. Biogenic Amines 23: 1-52

14. Ewing GW, Ewing EN, Parvez SH (2009) Developmental Dyslexia: the link with the Autonomic Nervous System and the Physiological Systems. J Biogenic Amines 23: 115-190

15. Ewing GW, Parvez SH. The influence of Pathologies and EEG frequencies upon sense perception and coordination in Developmental Dyslexia. A Unified Theory of Developmental Dyslexia. N Am J Med Sci: in press. 